\title{
INTRATYMPANIC LOW DOSE GENTAMICIN IN INTRACTABLE MENIERE'S DISEASE- CHEMICAL LABYRINTHECTOMY REVISITED
}

\author{
Ankit Choudhary1, Kaustuv Das Biswas², Bijan Basak³, Swapan Kumar Ghosh4, Dhiman Bhattacharya ${ }^{5}$ \\ ${ }^{1}$ Senior Resident, Department of ENT, Head and Neck Surgery, IPGMER \& SSKM Hospital, Kolkata, West Bengal, India. \\ ${ }^{2} R M O$ CUm Clinical Tutor, Department of ENT, Head and Neck Surgery, IPGMER \& SSKM Hospital, Kolkata, West Bengal, India. \\ ${ }^{3}$ Associate Professor, Department of ENT, Head and Neck Surgery, IPGMER \& SSKM Hospital, Kolkata, West Bengal, India. \\ 4 Professor, Department of ENT, Head and Neck Surgery, IPGMER \& SSKM Hospital, Kolkata, West Bengal, India. \\ ${ }^{5}$ Consultant Surgeon, Department of ENT, M. R. Bangur Hospital, Kolkata, West Bengal, India.
}

\section{ABSTRACT}

\section{BACKGROUND}

Meniere's disease is charecterised by episodic vertigo, fluctuating hearing loss and tinnitus. When medical treatment for Meniere's disease for treatment of vertigo fails i.e. when the vertigo becomes intractable, symptoms are disabling \& patients had been unresponsive to medical therapy for at least 1 year, we considered treating them by intratympanic gentamicin injection which is less invasive than surgical labyrinthectomy \& therefore more acceptable to some of the patients suffering from intractable vertigo.

\section{METHODS}

A prospective comparative study was conducted in the department of ENT from April 2017 to October 2018 on 32 patients of Meniere's disease having intractable vertigo not responding to conservative treatment. Of these patients, 16 patients were chosen randomly for intratympanic gentamicin injection and the remaining 16 patients acted as control, who were not given intratympanic gentamicin and treated with conservative management.

\section{RESULTS}

In this study complete vertigo control was found in $87.5 \%$ and substantial vertigo control was found in $12.5 \%$ of the patients. After treatment with intratympanic gentamicin, $12.5 \%$ of the patients showed a further sensorineural hearing loss audiologically.

\section{CONCLUSIONS}

From the findings of our study, it can be concluded that low dose intratympanic gentamicin administration is a safe, effective \& relatively simple method to control symptoms in patients with intractable Meniere's disease. A larger study is necessary to establish the dosing interval \& definite signs of improvement in disease control.

\section{KEY WORDS}

Intratympanic Gentamicin, Low Dose Gentamicin Protocol, Meniere's Disease, Vertigo

HOW TO CITE THIS ARTICLE: Choudhary A, Biswas KD, Basak B, et al. Intratympanic low dose gentamicin in intractable Meniere's disease- chemical labyrinthectomy revisited. J. Evolution Med. Dent. Sci. 2019;8(23):1843-1847, DOI: 10.14260/jemds/2019/405

\section{BACKGROUND}

Meniere's disease is characterised by episodic vertigo, fluctuating hearing loss and tinnitus and was first described by Prosper Meniere.

Cawthorne and Lindsay confirmed pathologically that Meniere's disease produces marked distension of endolymphatic labyrinth in the cochlea and vestibule.[1,2]

Knapp postulated that the distension was as a result of increased endolymphatic pressure, making a comparison to Meniere's disease with glaucoma.[3]

When medical treatment for Meniere's disease for treatment of vertigo fails i.e. when the vertigo becomes intractable, symptoms are disabling \& patients had been unresponsive to medical therapy for at least 1 year, the

'Financial or Other Competing Interest': None.

Submission 12-04-2019, Peer Review 24-05-2019,

Acceptance 01-06-2019, Published 10-06-2019.

Corresponding Author:

Dr. Kaustuv Das Biswas,

Avishikta-1, Flat 2B-402, 369/1,

Purbachal Kalitala Road,

P. O. Haltu, Kolkata-700078,

West Bengal, India.

E-mail: dr.kaustuv.82@gmail.com

DOI: $10.14260 /$ jemds $/ 2019 / 405$

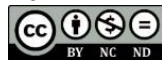

disease may be treated by intratympanic gentamicin injection as a last resort prior to surgical labyrinthectomy.

Intratympanic gentamicin treatment (ITG) for control of vertigo has become popular worldwide. In this treatment gentamicin solution is applied in the middle ear with the aim of damaging the vestibular hair cells with an intended result of a decrease (Or disappearing) of vertigo complaints while preserving hearing. ${ }^{[4-8]}$

By 1999, intratympanic gentamicin therapy (ITG) had become the most frequently used invasive treatment for Meniere's disease in USA.[9]

In this study, all the patients attending ENT OPD with features of Meniere's disease for the period from April 2017 to October, 2018 were managed with one year of conservative treatment. Patients with intractable vertigo, who did not respond to conservative treatment, were selected randomly for intratympanic gentamicin injection. A group acted as control whereas the other group was given the treatment.

The aim of this study was to study efficacy of intratympanic gentamicin as a treatment protocol in Meniere's disease with intractable vertigo, to compare the degree of sensorineural hearing loss in all treated patients with intratympanic gentamicin and to compare the collected data with available world literature. 


\section{METHODS}

Study Design

Prospective comparative study.

\section{Place of Study}

The study was conducted between April 2017 and October, 2018 in the department of ENT, IPGME \& R and SSKM Hospital, Kolkata. 32 patients of Meniere's disease were found having intractable vertigo not responding to conservative treatment and were selected for the study.

\section{Inclusion Criteria}

As per guidelines of American Association of Otolaryngologists and Head-Neck Surgeons (AAO-HNS, 1995) [10], the patients were diagnosed as definite Meniere's disease when they had two or more definitive spontaneous episodes of vertigo for 20 minutes or longer, audiologically documented sensorineural hearing loss (frequencies $0.5,1,2$, and $3 \mathrm{kHz}$ ) on at least one occasion, tinnitus or aural fullness in the affected ear and other causes being excluded. Meniere's disease patients with normal middle ear function, without systemic diseases like diabetes mellitus, bleeding disorders etc., who were never been given intratympanic gentamicin before and failed to respond to one-year medical therapy were selected for the study.

\section{Sampling Technique}

We used systematic Random sampling technique in our study. We numbered the patients who qualified for our study depending upon the inclusion criteria \& then used random number table to assign patients into intervention group \& control group.

\section{IEC Clearance}

Prior to commencement of the study institutional ethical committee clearance was sought for \& was obtained.

\section{Consent Process}

After selecting a patient for the intervention group informed consent was obtained \& documented from the patient. Information regarding the procedure, the expected outcome, possible side effects- all were passed on to the patients. Patients were free to consent for the intervention \& if any one did not give consent, he/she was put into the control group \& the next patient was considered for placement in intervention group.

\section{Rationale for Sampling Size}

As the study period was stipulated between April, 2017 and October, 2018, we considered all the patients of Meniere's disease attending to the OPD \& after imposing the inclusion \& exclusion criteria finally 32 patients were qualified for the study. So we divided them into two equal groups- 16 patients were chosen for intratympanic gentamicin and the remaining 16 patients acted as control who were treated conservatively and not given intratympanic gentamicin, thus making it possible to compare the results between the two groups.

Following investigations were done- routine blood tests $\mathrm{Hb} \%$, TC, DC, ESR, blood for sugar: fasting and post prandial, pure tone audiometry, impedance audiometry, caloric ENG, and HRCT of temporal bone (To exclude other causes of vertigo).
The selected cases were given intratympanic gentamicin injection therapy. Most of the cases responded with a single dose, 4 patients needed 2 consecutive doses and 2 patients were given 3 doses. The interval between 2 consecutive injections was 3 weeks.

The patients were followed up and final evaluation was done 6 months after the final dose of intratympanic gentamicin. Thus, in our study we followed the low dose protocol, giving 1-2 injections of gentamicin, with an interval of at least 3 weeks. The 2 nd injection is given only if there has been a vertigo spell in the 2 weeks prior. In other words, instead of titrating to the onset of damage to vestibular system (as is done for high-dose ITG), the criterion is a good effect on the disease control \& relief from symptoms.

The patient lies supine with the head tilted so that the ear to be given injection will be on the upper side. The intratympanic injection is done after application of $4 \%$ xylocaine in a cotton swab over the tympanic membrane for 15 minutes. $1 \mathrm{ml}$. of gentamicin solution in concentration of $20 \mathrm{mg} / \mathrm{ml}$. is injected through the tympanic membrane with a tuberculin syringe fitted with a small spinal needle. The spinal needle is bent so that the doctor can place the needle without having the syringe obscuring his vision. The solution is left in the middle ear for 30 minutes with the patient lying flat and not swallowing during this time.

Classification of control of vertigo was done according to AAO-HNS (1995).

- $\quad \mathrm{N}=($ ATTpost/ATTpre) multiplied by 100.

- $\quad$ ATTpre= number of attacks in 6 months period before first injection;

- $\quad$ ATTpost= number of attacks in 6 months period after last injection.

- Complete control- N (0) [Class A],

- Substantial control- N (1-40) [Class B],

- $\quad$ Limited control- N (41-80) [Class C],

- Insignificant control- N (81-120) [Class D],

- Worse control- N (>120) [Class E],

- When secondary treatment needed due to disability from vertigo- [Class F].

\section{RESULTS}

In this study, vertigo was completely improved in 6 out of 8 female patients $(75 \%)$ and in all male patients $(100 \%)$ (Table 1).

Vertigo was completely improved in all patients in age group of 22-40 and 41-58 years (100\%). In the age group of 59-74 years vertigo was completely improved in 1 out of 3 patients (33.33\%) (Table 2)

In this study vertigo was completely improved in $87.5 \%$ (class A) and substantially in $12.5 \%$ (class B) of the cases. No complete or substantial improvement was observed in the control cases (class E or F) (Table 3).

With use of intratympanic gentamicin as a treatment protocol, only $12.5 \%$ of the patients treated with gentamicin showed a further hearing loss audiometrically whereas the control group showed no hearing loss (Table 4).

This has got a 'p value' of 0.144 which is not statistically significant indicating that ototoxicity of intratympanic gentamicin is not significant. It also assures us about safety of the procedure. 


\begin{tabular}{|c|c|c|c|c|}
\hline \multirow{2}{*}{ Sex } & \multicolumn{2}{|c|}{ No. of Cases } & \multirow{2}{*}{ Total } & $\begin{array}{c}\text { Percentage of } \\
\text { Improvement }\end{array}$ \\
\cline { 2 - 3 } Improved & $\begin{array}{c}\text { Not } \\
\text { Improved }\end{array}$ & & $75 \%$ \\
\hline Female & 6 & 2 & 8 & $100 \%$ \\
\hline Male & 8 & 0 & 8 & Table 1. Control of Vertigo Among Treated Cases \\
\hline \multicolumn{4}{|r|}{ According to Sex Distribution } \\
\hline
\end{tabular}

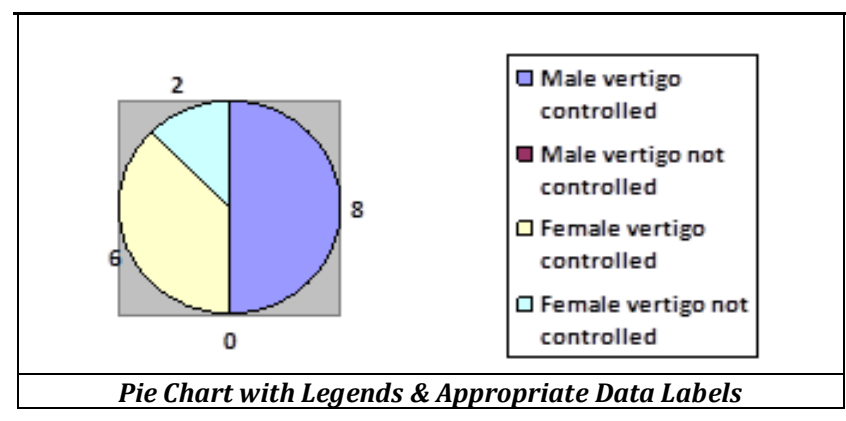

\begin{tabular}{|c|c|c|c|c|}
\hline $\begin{array}{c}\text { Age Group } \\
\text { in Years }\end{array}$ & Improved & $\begin{array}{c}\text { Not } \\
\text { Improved }\end{array}$ & Total & $\begin{array}{c}\text { Percentage of } \\
\text { Improvement }\end{array}$ \\
\hline $22-40$ & 11 & 0 & 11 & $100 \%$ \\
\hline $41-58$ & 2 & 0 & 2 & $100 \%$ \\
\hline $59-74$ & 1 & 2 & 3 & $33.33 \%$ \\
\hline Total & $\mathbf{1 4}$ & $\mathbf{2}$ & $\mathbf{1 6}$ & $\mathbf{8 7 . 5 \%}$ \\
\hline \multicolumn{6}{|c|}{ Table 2. Control of Vertigo According to Age Group } \\
\hline
\end{tabular}

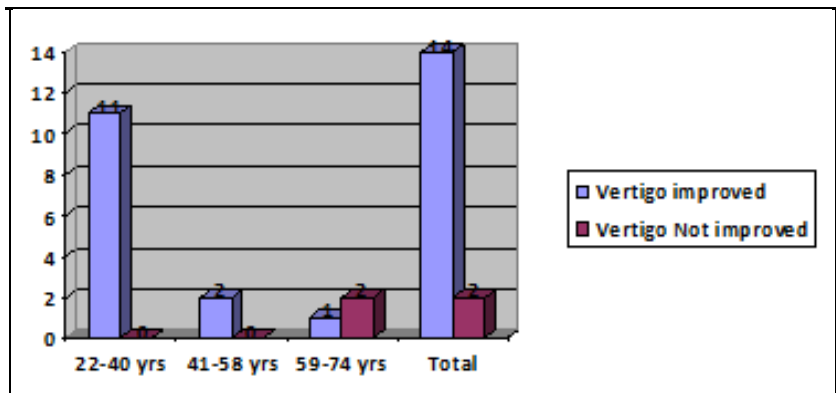

Figure 2. Depicting Treatment Results Across Various Age Groups

\begin{tabular}{|c|c|c|c|c|c|}
\hline $\begin{array}{c}\text { Vertigo } \\
\text { Control (Class) }\end{array}$ & Case & Percentage & $\begin{array}{c}\text { Control } \\
\text { Patients }\end{array}$ & Percentage & Total \\
\hline A & 14 & $87.5 \%$ & 0 & $0 \%$ & 14 \\
\hline B & 2 & $12 . \%$ & 0 & $0 \%$ & 2 \\
\hline E & 0 & $0 \%$ & 7 & $43.8 \%$ & 7 \\
\hline F & 0 & $0 \%$ & 9 & $56.3 \%$ & 9 \\
\hline Total & 16 & 16 & & 32 \\
\hline \multicolumn{7}{|c|}{ Table 3. Vertigo Control at 6 Months } \\
\hline
\end{tabular}

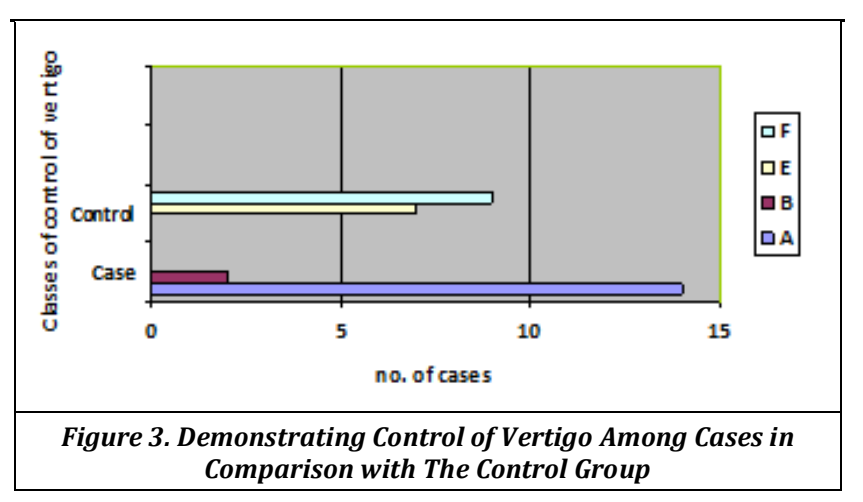

\begin{tabular}{|c|c|c|c|c|c|}
\hline & Case & Percentage & $\begin{array}{c}\text { Control } \\
\text { Patients }\end{array}$ & Percentage & Total \\
\hline $\begin{array}{c}\text { No Hearing } \\
\text { Loss }\end{array}$ & 14 & $87.5 \%$ & 16 & $100 \%$ & 30 \\
\hline Hearing Loss & 2 & $12.5 \%$ & 0 & $0 \%$ & 2 \\
\hline Total & $\mathbf{1 6}$ & \multicolumn{16}{|c|}{ Table 4. Further Hearing Loss After 6 Months } \\
\hline \multicolumn{7}{|c|}{}
\end{tabular}

\section{DISCUSSION}

The management of intractable vertigo in a case of Meniere's disease by intratympanic gentamicin injection has become popular because of the effective control of vertigo and minimal hearing loss, as gentamicin is more vestibulo-toxic than cochleo-toxic.[11]

It is thought that the gentamicin permeates the perilymph and the endolymph of the inner ear through the round and oval window membranes and damages the vestibular hair cells. However, the use of intratympanic gentamicin carries the risk of inducing hearing loss.[12] Aminoglycosides enter the cochlea rapidly following administration (Systemic and intratympanic) and reach a plateau within 0.5 to $3 \mathrm{~h}$ after application. Clearance from peri- and endolymph is slow, and aminoglycosides persist in the inner ear fluids and tissues long after the drug has been removed from the blood by renal clearance. The drugs may persist in the inner ear fluids for months after treatment and this may account for delayed hair cell degeneration.

In a study conducted by Mcfeely WJ et al in 1998 on 25 patients showed an improvement of vertigo in $88 \%$ of treated patients with intratympanic gentamicin.[13]

In another study conducted by Zhai $\mathrm{F}$ et al in 2010 showed an improvement of vertigo in $89 \%$ of patients and hearing loss in $16 \%$ of treated patients.[14]

In a study conducted by Delgardo LP et al in 2011 on 71 patients showed an improvement of vertigo in $84.37 \%$ of patients and hearing loss in $18.3 \%$ patients.[15]

De Beer L et al in a study on 57 patients in 2007 found an improvement of vertigo in $81 \%$ of patients (Class A, $61.4 \%$; class $\mathrm{B}, 19.3 \%$ ) and hearing loss (> $10 \mathrm{~dB}=\mathrm{SNHL}$ ) in $15.8 \%$ of patients. In their study ITG therapy was administered using a low dose of gentamicin (Approximately 21-24 mg per injection) applied at intervals of a minimum of 27 days. Only 1 injection was administered in $49.1 \%$ of the patients with improvement/ relief of symptoms \& the remaining $50.9 \%$ needed 2, 3, 4, 6 or 10 injections, always with a time interval of a minimum of 27 days.[16]

Atlas JT et al after a study on 68 patients in 1999 stated an improvement of vertigo in $84 \%$ and hearing loss in $17 \%$ of patients.[9]

Postema reported hearing loss over $15 \mathrm{~dB}$ in $25 \%$ of his patients in the active group compared to no hearing loss in the placebo group. One of these patients experienced $60 \mathrm{~dB}$ hearing loss. ${ }^{[17]}$ Stokroos stated no significant hearing loss in his study. The reason for this rather large difference could be that Stokroos used less gentamicin (1.5 injections versus four injections) and/ or had a larger time interval between two injections (six weeks versus one week) than Postema. ${ }^{[18]}$ In the present study, $12.5 \%$ of the patients showed a further hearing loss audiologically, corroborating with the previous studies.

There are several protocols for ITG, initially high dose protocol was used. In the high dose variant, more gentamicin is given over a shorter period of time. This variant works more often ( $90 \%$ of the time), but also is accompanied by far more risk. Injections are generally given every week, up to a total of 4-6. The treatment is stopped when vertigo ensues, indicating that the gentamicin is affecting the inner ear. In other words, the treatment is continued until there is evidence of damage to the inner ear. But later different modified \& low dose protocols have been advocated. In the 
study performed by Chia SH et al, a systemic meta-analysis was performed on different regimes of ITG therapy. Multiple daily dosing technique (Delivery three times per day for $=4$ d), weekly dosing technique (Weekly injections for four total doses), low-dose technique (One to two injections with retreatment for recurrent vertigo), continuous microcatheter delivery, and titration technique (Daily or weekly doses until onset of vestibular symptoms, change in vertigo, or hearing loss) were compared. They concluded that the titration method of gentamicin delivery demonstrated significantly better complete $(81.7 \%, \mathrm{p}=0.001)$ and effective $(96.3 \%, \mathrm{p}<$ $0.05)$ vertigo control compared with other methods. They also noted that the weekly method of delivery tends toward less overall hearing loss $(13.1 \%, p=0.08)$, and the multiple daily method demonstrated significantly more overall hearing loss $(34.7 \%, \mathrm{p}<0.01)$ compared with other groups.[19]

Steven D et al reported the result of ITG therapy in patients with intractable Meniere's disease \& they found complete control of episodic vertigo initially in 20 of 21 patients $(95.2 \%)$. However, 6 of 20 responders $(30 \%)$ developed relapsing symptoms within 12 months. Retreatment was successful in $75 \%$ of these patients. Overall, hearing was preserved or improved in $62 \%$ of cases, worse in $24 \%$, and not yet tested in $14 \%$. When the cumulative dose of gentamicin was $\leq 4$ injections in the first week, only 1 of 14 (7.1\%) lost hearing. Thus, they concluded that intratympanic gentamicin offers better risk/benefit outcome than other invasive therapies for intractable Meniere's disease. [20]

According to the study performed by Perez $\mathrm{N}$ et al in 2003 , Vertigo was controlled in $83.1 \%$ of the 71 patients. Recurrence of vertigo after initially complete control was noted in 17 patients. In 13 of these patients, this was cured by another course of intratympanic injections of gentamicin. Hearing level as pure-tone average was unchanged 2 years after treatment, but hearing loss as a result of gentamicin injections occurred in 23 patients at the end of treatment and in 9 and 11 patients at 3 months and 2 years after the treatment, respectively. Thus, their findings are closely resembling those found in the present study regarding control of vertigo. So from the findings of their study, Perez et al concluded that, ending weekly intratympanic injections when clinical signs of vestibular deafferentation appear can control vertigo in the majority of patients, and it is a useful alternative, together with other surgical options, for the treatment of patients with Meniere's disease who do not respond to medical treatment.[21]

Similar results were reflected by Gilony D et al in 2004, who reported that following treatment, 20 (87\%) patients no longer experienced vertigo attacks, while 16 of them $(69.6 \%)$ reported full functional recovery. Two patients $(8.7 \%)$ did not respond to treatment, and therefore, underwent surgical procedures. One patient (4.4\%) did not complete therapy. The mean change in hearing examination was non-significant. However, two of the 21 patients suffered a change from moderate to severe hearing loss to complete deafness (9.5\%). There was no case of post treatment infection or permanent tympanic perforation.

Thus, the authors commented that, Intratympanic gentamicin injection is a simple and safe procedure for intractable Meniere's patients, with a high success rate and low risk for hearing deterioration. It is recommended for patients with continuous functional disability prior to surgical treatment.[22]

In a recent study published by Veronica $\mathrm{R}$ et al. in 2013, it was possible to achieve complete control of vertigo attacks in $60 \%$ (Class A) of the patients and effective control in the $88 \%$ (classes A and B). 16\% of the cases needed two cycles of treatment in order to obtain these results. Regarding hearing outcome PTA remained unchanged in 18 patients $(72 \%)$; it improved in 4 patients (16\%; maximum $17,5 \mathrm{~dB}$ ); and it worsened in 3 patients (12\%; maximum $18,7 \mathrm{~dB})$. With regard to patients who received a second cycle of treatment, PTA did not change in 3 patients (75\%) and it worsened in 1 patient $(25 \%)$ after the second cycle. In view of the results, they concluded that gentamicin administration for intractable Meniere's disease is a relatively safe and effective treatment for the control of vertigo attacks.[23]

In connection with mechanism of action of gentamicin, Minor LB pointed out that Gentamicin may relieve vertigo due to its toxic effects on the dark cells and the stria vascularis of the labyrinth without causing any changes in the vestibular function. [24]

In the present study the patients were followed up for 6 months after the final dose of intratympanic gentamicin injection. According to age group, only 1 out of 3 patients showed complete improvement of vertigo in older age group (59-74 years), but in younger age group (22-40 and 41-58 years) all patients had complete improvement of vertigo. Overall improvement of vertigo was in $87.5 \%$ of patients in category A range (Complete) and $12.5 \%$ of patients in category B range (Substantial), which almost correlates with the work done by previous authors. The 'p value' here is $<.0001$ which is statistically significant; i.e. it signifies intratympanic gentamicin as a statistically proven treatment policy for intractable vertigo in a case of Meniere's disease.

With use of intratympanic gentamicin as a treatment protocol, only $12.5 \%$ of the patients treated with gentamicin showed a further hearing loss audiometrically whereas the control group showed no hearing loss. This has got a ' $p$ value' of 0.144 which is not statistically significant indicating that ototoxicity of intratympanic gentamicin is not significant. Thus, the procedure of intratympanic gentamicin appears safe \& effective in symptom control without incurring any significant hearing handicap to the patients, thus ultimately improving the quality of life of the patients.

\section{CONCLUSIONS}

Low dose intratympanic gentamicin administration is a safe, effective \& relatively simple method to control symptoms in patients with intractable Meniere's disease. However, a larger study is necessary to establish the dosing interval \& definite signs of improvement in disease control.

\section{REFERENCES}

[1] Cawthorne TE. Meniere's disease. Ann Otol Rhinol Laryngol 1947;56(1):18-38.

[2] Lindsay JR. Labyrinthine dropsy. Laryngoscope 1946;56:325-41.

[3] Knapp H. A clinical analysis of inflammatory affections of the inner ear. Arch Ophthalmol 1871;2:202-83.

[4] Minor LB, Schessel DA, Carey JP. Ménière's disease. Curr Opin Neurol 2004;17(1):9-16. 
[5] Blakley BW. Clinical forum: a review of intratympanic therapy. Am J Otol 1997;18(4):520-31.

[6] Pender DJ. Gentamicin tympanoclysis: effects on the labyrinthine sensory cells. Laryngoscope 2003;113(2):343-8.

[7] Lii M, Ding D, Zheng XY, et al. Vestibular destruction by slow infusion of gentamicin into semicircular canals. Acta Otolaryngol Suppl 2004;(552):35-41.

[8] Pullens B, van Benthem PP. Intratympanic gentamicin for Ménière's disease or syndrome. Cochrane Database Syst Rev 2011;(3):CD008234.

[9] Atlas JT, Parnes LS. Intratympanic gentamicin titration therapy for Meniere's disease. Am J Otol 1999;20(3):357-63.

[10] Committee on Hearing and Equilibrium Guidelines for the diagnosis and evaluation of therapy in Meniere's disease. American Academy of Otolaryngology- Head and Neck Foundation, Inc. Otolaryngology - Head and Neck Surgery 1995;113(3):181-5.

[11] Bremer HG, de Groot JC, Versnel H, et al. Combined administration of kanamycin and furosemide does not result in loss of vestibular function in Guinea pigs. Audiol Neurootol 2012;17(1):25-38.

[12] Carey J. Intratympanic gentamicin for the treatment of Meniere's disease and other forms of peripheral vertigo. Otolaryngol Clin North Am 2004;37(5):107590.

[13] McFeely WJ, Singleton GT, Rodriguez FJ, et al. Intratympanic gentamicin injection in Meniere's disease. Otolaryngol Head Neck Surg 1998;118:58996.

[14] Zhai F, Liu JP, Dai CF, et al. Evidence-based modification of intratympanic gentamicin injections in patients with intractable vertigo. Otol Neurotol 2010;31(4):642-8.
[15] Delgardo LP, Rodrigo JF, Pena PA. Intratympanic gentamicin in Meniere's disease: our experience. J Laryngol Otol 2011;125(4):363-9.

[16] De Beer L, Stokroos R, Kingma H. Intratympanic gentamicin therapy for intractable Meniere's disease. Acta Otolaryngol 2007;127(Suppl 6):605-12.

[17] Postema RJ, Kingma CM, Wit HP, et al. Intratympanic gentamicin therapy for control of vertigo in unilateral Meniere's disease: a prospective, double-blind, randomized, placebo-controlled trail. Acta OtoLaryngologica 2008;128(8):876-80.

[18] Stokroos R, Kingma H. Selective vestibular ablation by intratympanic gentamicin in patients with unilateral active Meniere's disease: a prospective, double-blind, placebo-controlled, randomized clinical trial. Acta OtoLaryngologica 2004;124(2):172-5.

[19] Chia SH, Gamst AC, Anderson JP, et al. Intratympanic gentamicin therapy for Ménière's disease: a metaanalysis. Otol Neurotol 2004;25(4):544-52.

[20] Rauch SD, Oas JG. Intratympanic gentamicin for treatment of intractable Meniere's disease: a preliminary report. Laryngoscope 1997;107(1):49-55.

[21] Perez N, Martín E, García-Tapia R. Intratympanic gentamicin for intractable Meniere's disease. Laryngoscope 2003;113(3):456-64.

[22] Gilony D, Wolf M, Kronenberg J. Intratympanic gentamicin treatment for intractable Menière's disease. Harefuah 2004;143(1):4-7, 88.

[23] Verónica R, Herminio PG, Constantino M. Evaluation of two procedures of intratympanic gentamicin therapy in Meniere's disease. Int Adv Otol 2013;9(1):82-8.

[24] Minor LB. Intratiympanic gentamicin for control of vertigo in Meniere's disease: vestibular signs that specify completion of therapy. Am J Otol 1999;20 (2):209-19. 